\title{
GÜNCEL ÇOK KRITTERLİ KARAR VERME TEKNIKLERI
}

\author{
Dr. Gülay DEMİR \\ Prof. Dr. Mahmut KARTAL
}




\section{(C) Copyright 2020}

Bu kitabın, basım, yayın ve satış hakları Akademisyen Kitabevi A.Ş.'ne aittir. Anılan kuruluşun izni alınmadan kitabın tümü ya da bölümleri mekanik, elektronik, fotokopi, manyetik kağıt ve/veya başka yöntemlerle çoğaltılamaz, basılamaz, dağıtılamaz. Tablo, şekil ve grafikler izin alınmadan, ticari amaçh kullanılamaz. Bu kitap T.C. Kültür Bakanlı̆̆ bandrolü ile satılmaktadır.

Bu kitap Prof. Dr. Mahmut KARTAL danışmanlığında, Gülay Demir tarafından hazırlanan "Çok Kriterli Karar Verme Teknikleri ile Aynı Dilimdeki Fen Liselerinin Değerlendirilmesi” isimli doktora tezinden türetilmiştir.

ISBN

978-605-258-936-6

\section{Kitap Adı}

Güncel Çok Kriterli Karar Verme Teknikleri

Yazarlar

Gülay DEMİR

Mahmut KARTAL

Yayın Koordinatörü

Yasin Dilmen

Sayfa ve Kapak Tasarımı

Akademisyen Dizgi Ünitesi

Yayıncı Sertifika No

25465

\section{Baskı ve Cilt}

Sonçağ Matbaacılık

Bisac Code

BUS092000

\section{GENEL DAĞITIM}

\section{Akademisyen Kitabevi A.Ş.}

Halk Sokak 5 / A

Yenişehir / Ankara

Tel: o312 4311633

siparis@akademisyen.com

\section{www . a ka de misyen.com}




\section{ÖNSÖZ}

Karar verme, mevcut durumları değerlendirerek bir amaca ulaşmak için çeşitli faaliyetlerden en uygun olanı seçme işlemidir. Uzmanların bakış açısıyla karar verme, problemi tanımlama, olası çözümleri oluşturma, kriterleri seçme, her çözümün sonuçlarını belirleme, çözümleri değerlendirme ve en iyi çözümü seçme gibi farklı aşamalara sahiptir. Karar verme işlemi bir süreçtir. $\mathrm{Bu}$ süreci kolaylaştırmak için çok sayıda kriter ile karar verme (ÇKKV) teknikleri geliştirilmiştir. Bu teknikler birden fazla kriterin olduğu durumlarda karar vericinin karar sonucunu mümkün olduğunca hızlı ve basit elde etmesi amacıyla kullanılır. ÇKKV, karar verme tekniklerinin toplamıdır. Günümüzde bu önemli ve uygulamalı teknikler ile ilgili birçok makale, kitap ve tez yayınlanmıştır. Bu konuda bilimsel ve araştırma çalışmaları devam etmektedir.

ÇKKV, en iyi çözümü tasarlamak için Çok Amaçlı Karar Verme $($ ÇAKV) $=$ Multi Objective Decision Making (MODM) ve en iyi alternatifi seçmek için Çok Nitelikli Karar Verme (ÇNKV) =Multi Attribute Decision Making (MADM) olarak ikiye ayr1lır. ÇNKV uygulamaları ÇAKV uygulamalarından daha fazladır. Bazı kaynaklar ÇKKV ile ÇNKV aynı kavram olarak kullandığından kitapda aynı tutum izlenmiştir.

Mevcut kitapta, güncel 14 ÇKKV tekniği ayrıntılı olarak tanıtılmıştır. Kitabın her bölümünde, yöntemin adımları açıklanmış ve tekniğe bir örnek sunulmuştur.

Kitabın yayın ve dağıtım süreçlerini üstlenen Akademisyen Yayıncılık yöneticilerine ve çalışanlarına özverili tutumları nedeniyle teşekkür ederim. 
Kitabın okuyucular, araştırmacılar ve yöneticiler için yararlı olmasinı dilerim.

Sivas, 2020

Dr. Gülay DEMİR 


\section{iÇiNDEKILER}

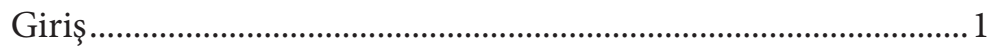

\section{BÖLÜM}

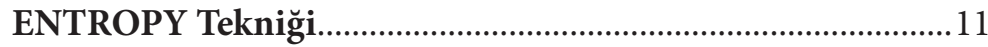

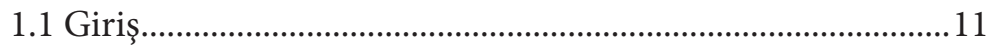

1.2 ENTROPY Tekniğinin Uygulama Adımları...........................11

1.3 ENTROPY Tekniğinin Örnek Uygulaması ............................ 12

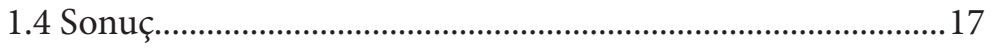

\section{BÖLÜM}

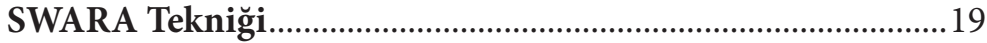

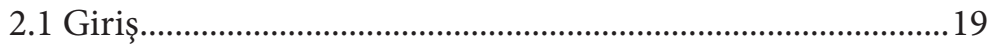

2.2 SWARA Tekniğinin Uygulama Adımları.................................19

2.3 SWARA Tekniğinin Örnek Uygulaması....................................21

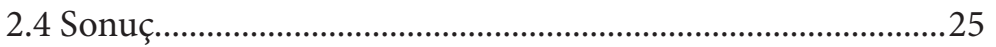

\section{BÖLÜM}

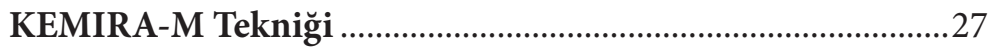

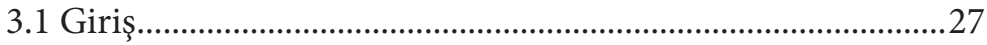

3.2 KEMIRA-M Tekniğinin Uygulama Adımları ........................27

3.3 KEMIRA-M Tekniğinin Örnek Uygulamas1 ...........................30

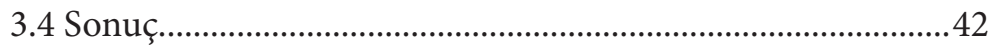

\section{BÖLÜM}

CRITIC Tekniği ..................................................................43

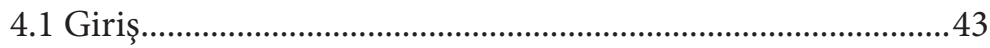

4.2 CRITIC Tekniğinin Uygulama Adımları .................................43

4.3 CRITIC Tekniğinin Örnek Uygulaması ...................................4

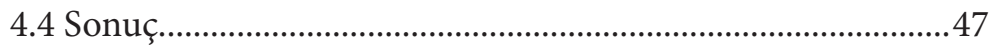




\section{BÖLÜM}

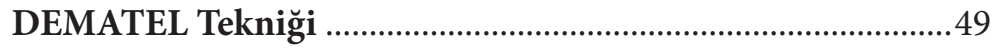

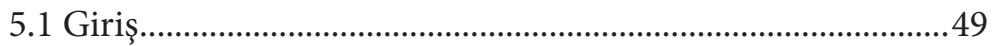

5.2. DEMATEL Tekniğinin Uygulama Adımları .........................49

5.3 DEMATEL Tekniğinin Örnek Uygulaması ...............................51

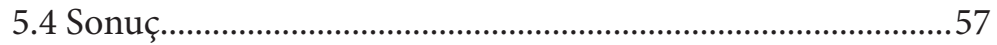

\section{BÖLÜM}

\section{ARAS Tekniği}

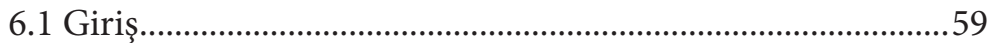

6.2 ARAS Tekniğinin Uygulama Adımları ...................................59

6.3 ARAS Tekniğinin Örnek Uygulamas1 .......................................61

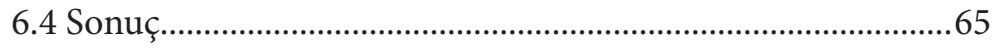

\section{BÖLÜM}

\section{WASPAS Tekniği}

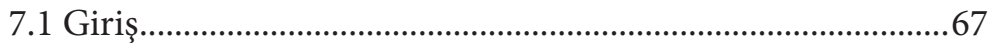

7.2 WASPAS Tekniğinin Uygulama Adımları ..............................67

7.3 WASPAS Tekniğinin Örnek Uygulamas1 .................................69

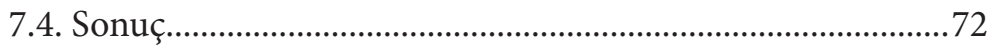

\section{BÖLÜM}

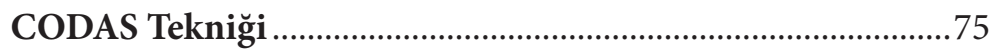

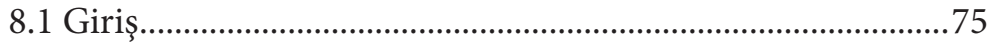

8.2. CODAS Tekniğinin Uygulama Adımları .................................75

8.3 CODAS Tekniğinin Örnek Uygulaması....................................77

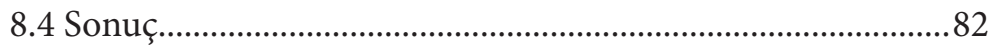

\section{BÖLÜM}

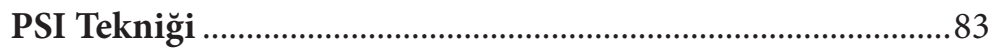


9.1 Giriş. 83

9.2 PSI Tekniğginin Uygulama Adımları ...................................83

9.3. PSI Tekniğinin Örnek Uygulaması ......................................85

9.4 Sonuç 88

\section{BÖLÜM}

\section{MABAC Tekniği}

10.1 Giriş...... .89

10.2 MABAC Tekniğinin Uygulama Adımları 89

10.3 MABAC Tekniğinin Örnek Uygulaması.................................92

10.4 Sonuç 96

\section{BÖLÜM}

\section{MAIRCA Tekniği}

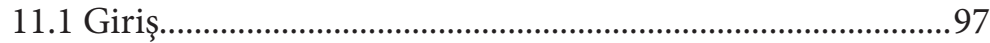

11.2 MAIRCA Tekniğinin Uygulama Adımları .........................97

11.3 MAIRCA Tekniğinin Örnek Uygulaması .............................99

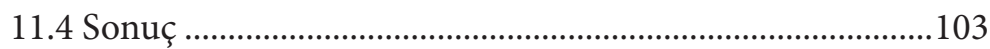

\section{BÖLÜM}

\section{MOOSRA Tekniği}

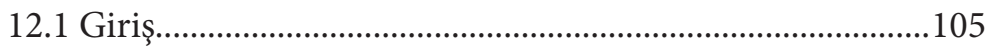

12.2 MOOSRA Tekniğinin Uygulama Adımları........................105

12.3 MOOSRA Tekniğinin Örnek Uygulaması............................106

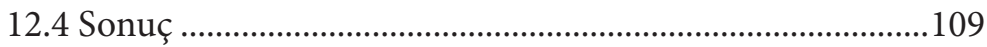

\section{BÖLÜM}

EDAS Tekniği

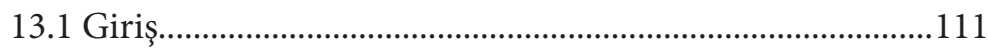

13.2 EDAS Tekniğinin Uygulama Adımları................................111

13.3 EDAS Tekniğinin Örnek Uygulaması ...................................113 


\section{BÖLÜM}

COPELAND Tekniği

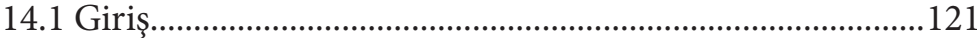

14.2 COPELAND Tekniğinin Uygulama Adımları ....................121

14.3 COPELAND Tekniğinin Örnek Uygulaması ......................123

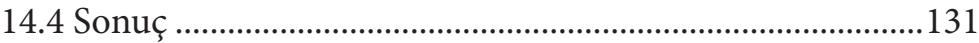

\section{BÖLÜM}

Duyarlılık Analizi

\section{BÖLÜM}

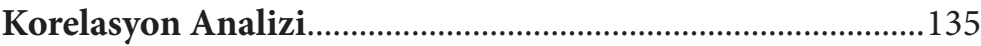

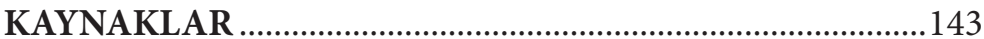




\section{KAYNAKLAR}

Andreica Mădălina Ecaterina, Dobre Ion, Andreica Mugurel Ionuț ve Resteanu Cornel (2010). "A New Portfolio Selection Method Based on Interval Data". Studies in Informatics and Control. 19(3): 253-262.

Asr Tajvidi, Hayaty M, Rafiee Ramin, Ataie Mohammed ve Jalalı Seyyed Mohammad Esmaeil (2015). "Selection of Optimum Tunnel Support System Using Aggregated Ranking of SAW, TOPSIS and LA Methods". International Journal of Applied Operational Research. 5(4): 49-63.

Belton Valerie ve Stewart Theo (2003). Multiple Criteria Decision Analysis An Integrated Approach. USA: Kluwer Academic Publishers.

Chan Kai MA, Satterfield Terre ve Goldstein Jashua (2012). "Rethinking Ecosystem Services

Das Manik Candra, Sarkar Bijan, ve Ray Siddhartha (2012). "Decision Making Under Conflicting Environment: A New MCDM Method". International Journal of Applied Decision Sciences. 5(2): 142-162.

Diakoulaki Danae, Mavrotas George ve Papayannakis Lefteris (1995). "Determining Objective Weights in Multiple Criteria Problems: The CRITIC Method". Computers and Operation Research. 22(79): 763-770.

Fontela, Emilio ve Gabus Andre (1976). "The DEMATEL Observer". Battle Institute, Geneva Research Center. 56-61.

Ghorabaee Mehdi Keshavarz, Amiri Maghsoud, Zavadskas Edmundas Kazimieras, Hooshmand Reyhaneh ve Antucheviciene Jurgita (2017). "Fuzzy Extension of the CODAS Method for Multi Criteria Market Segment Evaluation". Journal of Business Economics and Management.18(1): 1-19.

Ghorabaee Mehdi Keshavarz, Zavadskas Edmundas Kazimieras, Olfat Laya ve Turskis Zenonas (2015). "Multi Criteria Inventory Classification Using A New Method of Evaluation Based on Distance from Average Solution (EDAS)". Informatica. 26(3): 435-451.

Gigović Ljubomir, Pamučar Dragan, Bajić Zoran ve Milićević Milić (2016). "The Combination of Expert Judgment and GIS-MAIRCA Analysis for the Selection of Sites for Ammunition Depots". Sustainability. 8(4): 2-30.

Huang Chi-Yo, Joseph Z. Shyu ve Tzeng Gwo-Hshiung (2007). "Reconfiguring the Innovation Policy Portfolios for Taiwan's SIP Mall Industry", Technovation, 27(12): 744-765.

Jahan Ali, Mustapha Faizal, Sapuan S. M, Ismail M. Yusof ve Bahraminasab Marjan (2012). "A Framework for Weighting of Criteria 
in Ranking Stage of Material Selection Process". The International Journal of Advenced Manufacturing Technology, 58(1): 411-420.

Janssen, Ron (2001). "On the Use of Multi-Criteria Analysisin Environmental Impact Assessment in the Netherlands". Journal of Multi-Criteria Decision Analysis. 10: 101-109.

Kashi Katerina (2015). "DEMATEL Method in Practice: Finding the Causal Relations Among Key Comperencie", The $9^{\text {th }}$ International Days of Statistics and Economics, Prague: 723-732.

Keršuliene Violeta, Zavadskas Edmundas Kazimieras ve Turskis Zenonas (2010). "Selection of Rational Dispute Resolution Method by Applying New Stepwise Weight Assessment Ratio Analysis (SWARA)". Journal of Business Economics and Management. 11(2): 243258.

Keune Hans ve Dendencker Nicolas (2013). Negatiated Complexity in Ecosystem Services Science and Policy Making-Ecosystem Services: In Ecosystem Services-Global Issues Local Practices. New York: Elsevier.

Krylovas Aleksandras, Zavadskas Edmundas Kazimieras ve Kosareva Natalja (2016) "Multiple Criteria Decision Making KEMIRA-M Method for Solution of Location Alternatives". Economic Research. 29(1): 50-65.

Krylovas Aleksandras, Zavadskas Edmundas Kazimieras, Kosareva Natalja ve Dadelo Stanislav (2014). "New KEMIRA Method for Determining Criteria Priority and Weights in Solving MCDM Problem". International Journal of Information Technology and Decision Making.13:1119-1133.

Lashgari Shima, Antuchevičienė Jurgita, Delavari Alireza ve Kheirkhah Omid (2014). "Using QSPM and WASPAS Methods for Determining Outsourcing Strategies". Journal of Business Economics and Management. 15(4): 729-743.

Li Chung-Wei ve Tzeng Gwo-Hshiung (2009). "Identification of A Threshold Value For The DEMATEL Method Using The Maximum Mean Entropy Algorithm to Find Critical Services Provided by A Semiconducter Intellectual Property Mall", Expert Systems with Applications, 36(6): 9891-9898.

Locatelli Bruno, Rojas Varinia ve Salinas Zenia (2008). "Impact of Payments for Environmental Services on local Development in Nortern Costa Rica: A Fuzzy Multi-Criteria Analysis". Forest Policy and Economics. 10(5): 275-285.

Madic Milos, Gecevska Valentina, Radovanovic Miroslav ve Petkovic Dusan (2014). "Multi-Criteria Economic Analysis of Machining 
Processes Using the WASPAS Method". Journal of Production Engineering. 17(2): 79-82.

Majumder, Mrinmoy (2015). "Impact of Urbanization on Water Shortage in Face of Climatic Aberration". Springer Briefs in Water Science and Technology. 22: 30-39.

Maniya Kalpesh ve Bhatt Mangal G. (2010). "A Selection of Material Using A Novel Type Decision Making Method: Preference Selection Index Method". Materials and Design. 31(4):1785-1789.

Mendoza Guillermo A. ve Martins Helena (2006). "Multicriteria Decision Analysis in Natura Resource Management: A Critical Review of Methods and New Modeling Paradigms". Forest Ecology and Management. 230: 1-22.

Milani Abbas, Shanian Ali ve Madoliat Reza (2005). “The Effect of Normalization Norms in Multiple Attribute Decision Making Models: A Case Study in Gear Material Selection". Structural Multidisciplinary Optimization. 29(4): 312-318.

Milosavljevića Milan, Bursaća Marko ve Tričkovića Goran (2018). “Selection of the Railroad Container Terminal in Serbia Based on Multi Criteria Decision-Making Methods". Decision Making: Applications in Management and Engineering. 1(2): 1-15.

Munda Giuseppe, Nijkamp Peter ve Rietveld Piet (1994). "Qualitative Multicriteria Evaluation for Environmental Management". Ecological Economics. 10: 97-112.

Nilashi Mehrbakhsh, Zakaria Rozana, Ibrahim Othman, Majid Muhd Zaimi Abd, Rosli Mohamed Zin ve Farahmand Mohammadali (2015). "MCPCM: A DEMATEL-ANP Based Multi Criteria Decision Making Approach to Evaluate The Critical Success Factors in Construction Projects", Arab. J. Sci. Eng. 40(2): 343-361.

Özçalıc1, Meltem (2017). MATLAB ile Çok Kriterli Karar Verme Teknikleri. Ankara: Nobel Yayıncilık.

Pamučar Dragan ve Ćirović Goran (2015). “The Selection of Transport and Handling Resources in Logistics Centers Using Multi-Attributive Border Approximation Area Comparison (MABAC)". Expert Systems with Applications. 42(6): 3016- 3028.

Pourjavad Ehsan ve Shirouyehzad Hadi (2011). "A MCDM Approach for Prioritizing Production Lines: A Case Study". International Journal of Business and Management. 6(10): 221-229.

Pöyhönen Mari ve Hämäläinen Ramio (2001). "On the Convergence of Multi Attribute Weighting Methods”. European Journal of Operational Research. 129(3): 569-583. 
Saari Donald ve Merlin Vincent (1996). "The Copeland Method", Economic Theory. 8: 51-76.

Sarıçalı, Gizem (2018). "Çok Kriterli Karar Verme Yöntemlerinden KEMIRA-M ve COPRAS Yöntemlerinin Mermer İşletmesinde Makine Seçim Sürecine Uygulanması". Pamukkale Üniversitesi, Sosyal Bilimler Enstitüsü, Yüksek Lisans Tezi. Denizli.

Sarıçalı Gizem ve Kundakcı Nilsen (2017). "Forklift Alternatiflerinin KEMIRA-M Yöntemi ile Değerlendirilmesi”. Optimum Ekonomi ve Yönetim Bilimleri Dergisi. 4(1): 35-53.

Seyed-Hosseini Seyed Mohammad, Safaei Nima ve Asgharpour Mohammad Javad (2006). "Reprioritization of Failures in A System Failure Mode and Effects Analysis by Decision Making Trial and Evaluation Laboratory Technique", Reliability Engineering and System Safety, 91(8): 872-881.

Shemshadi Ali, Shirazi Hossein, Toreihi Mehran ve Tarokh Mohammad Jafar (2011). "A Fuzzy VIKOR Method for Supplier Selection Based on Entropy Measure for Objective Weighting". Expert System With Applications. 38(10): 12160-12167.

Shih Shushih, Shyur Huan Jhy ve Lee Stanley (2007). "An Extension of TOPSIS for Group Decision Making." Mathematical and Computer Modelling. 45: 801-813.

Stanujkić Dragisa, Karabašević Darjan ve Zavadskas Edmundas Kazimieras (2015). "A Framework for the Selection of A Packaging Design Based on the SWARA Method". Engineering Economics. 26(2): 181-187.

Timor, Mehpare (2011). Analitik Hiyerarşi Prosesi. İstanbul: Türkmen Kitabevi.

Toktaş Pelin ve Can Gülin Feryal (2018). "Şantiyelerin İş Sağlığı ve Güvenliği Açısından Risk Düzeylerine Göre KEMIRA-M Yöntemi ile Siralanmasi". Ergonomi. 1(3): 123-136.

Triantaphyllou Evangelos (2000). Multi-Criteria Decision Making Methods: A Comparative Study. Netherlands: Kluwer Academic Publishers.

Tseng Ming-Lang ve Lin Yuan Hsu (2008). "Application of Fuzzy DEMATEL to Develop A Cause And Effect Model Of Municipal Solid Waste". Environmental Monitoring and Assessment. 01-2: 158-171.

Wang Tien-Chin ve Lee Hsien Daa (2009). "Developing a Fuzzy TOPSIS Approach Based on Subjective Weights and Objective Weights". Expert Systems with Entropy Applications. 36(5): 8980-8985. 
Wu Jie, Sun Jiasen, Liang Liang ve Zha Yingchun (2011). "Determination of Weights For Ultimate Cross Efficiency Using Shannon Entropy". Expert Systems With Applications. 38(5): 5162-5165.

Wu Wei-Wen (2008). "Choosing Knowledge Management Strategies by Using A Combined ANP and DEMATEL Approach". Expert Systems With Applications. 35(3): 828-835.

Yoon Paul ve Hwang Ching-Lai (1995). "Multiple Attribute Decision Making: An Introduction". Sage Pub. Thousand Oaks. 6: 13-15.

Zardari Nour Hassan, Ahmed Kamal, Shirazi Sharif Moniruzzaman ve Yusop Zulkifli Bin (2015). Weighting Methods and Their Effect on Multi-Criteria Decision Making Model Outcomes in Water Resources Management. London: Springer.

Zavadskas Edmundas Kazimieras, Turskis Zenonas, Antucheviciene Jurgita ve Zakarevicius Algimantas (2012). "Optimization of Weighted Aggregated Sum Product Assessment”. Elektronika ir elektrotechnika. 122(6): 3-6.

Zavadskas Edmundas Kazimieras, Turskis Zenonas ve Vilutiene Tatjana (2010). "Multiple Criteria Analysis of Foundation Instalment Alternatives by Applying Additive Ratio Assessment (ARAS) Method". Archives of Civil and Mechanical Engineering. 10(3): 123-141.

Zolfani Sarfaraz Hashemkhani ve Saparauskas Jonas (2013). "New Application of SWARA Method in Prioritizing Sustainability Assessment Indicators of Energy System". Engineering Economics. 24(5): 408-414. 